\title{
Application of 3D laser scanning technology in engineering field
}

\author{
Chang'an $\mathrm{Hu}^{1, *}$, Linghui Kong ${ }^{1}$, Fei $\mathrm{Lv}^{2}$ \\ ${ }^{1}$ National Institute of Measurement and Testing Technology, 610021, Chengdu, China \\ ${ }^{2}$ Chengdu Normal University, 611130, Chengdu, China
}

\begin{abstract}
With the continuous progress of computer and laser measurement technology, non-contact measurement based on laser scanning technology has been more and more applied in the industrial production process. Since the 1990s, 3D laser scanning technology used as a new technology has been developed rapidly. The 3D laser scanning technology, also known as High Definition Surveying (HDS), was based on the principle of laser ranging. The technology on the surface of a measured object can record some features of large dense points, including $3 \mathrm{~d}$ coordinates, reflectance and texture information, so as to rapidly reconstruct 3D models of a measured target and various map data such as the line, the surface and the body. Meanwhile, it is a revolutionary leap that the technology improves the traditional single point measurement to the surface measurement, and realizes the digital reconstruction of the objects. Especially, for some conditions such as contact measurement and complex component detection that cannot be measured, 3D laser scanning technology has a natural advantage in the field of industrial design and measurement .
\end{abstract}

\section{Introduction}

With the development of all kinds of engineering applications, people are eager to meet the requirements of new mapping technology in complex sites and space. However, the traditional single point mapping technology can only obtain a single or some discrete points of $3 \mathrm{~d}$ coordinate data. When it is necessary to map the surface and entity of complex structure, a large number of mapping points need to be collected for single point mapping to ensure the integrity of the target structure, so the speed is slow and inefficient, and the modeling process is very complicated ${ }^{[1]}$.

Since the mid-1990s, more and more attention has been paid to a mapping technique called 3D laser scanning. The 3D laser scanner uses the principle of laser ranging, which records the $3 \mathrm{D}$ coordinates, reflectivity and texture of a large number of dense points on the surface of the object being measured. It can quickly reconstruct the 3D model of the object to be measured and various map data such as line, surface, system, etc. Compared with traditional single point measurement, 3D laser scanning technology is called as a revolutionary technology from single point measurement to surface measurement, which can complete the digital reconstruction of real objects. At present, more and more kinds of 3D laser scanners are used in the fields of cultural heritage protection, architecture, planning, civil engineering, factory renovation, interior design, building monitoring, traffic accident handling, legal evidence collection, disaster assessment, ship design, digital city, military analysis and so on.

\section{3D laser scanner}

\subsection{Composition}

Three-dimensional laser scanner is composed of laser scanner, digital camera, scanner rotation platform, software control platform, data processing platform, power supply and other accessories ${ }^{[2]}$. It is a new spatial information data acquisition method that integrates a variety of high and new technologies, as shown in Figure 1. Using $3 \mathrm{~d}$ laser scanning technology, can go deep into the scan in any complex scene environment and space operations, and can directly implement all kinds of large, complex and irregular, standard or non-standard entity or the complete $3 \mathrm{~d}$ data acquisition of real and rapid reconstruction of a physical object $3 \mathrm{~d}$ model and line, face, body, space, etc. all kinds of mapping data. At the same time, the collected 3D laser point cloud data can be used for various post-processing and analysis, such as mapping, measurement, analysis, simulation, display, monitoring, virtual reality and other operations. The collected 3D point cloud data and 3D modeling results can be converted into standard format and output into file format that can be recognized and processed by other engineering software.

\footnotetext{
* Corresponding author: 569964114@qq.com
} 


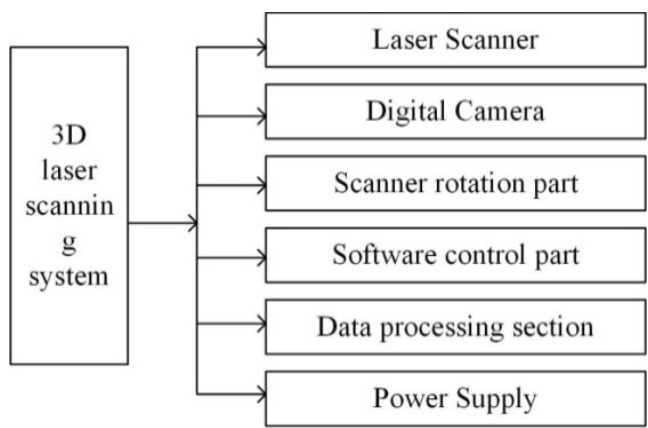

FIG. 1 Composition of $3 \mathrm{~d}$ laser scanning system

\subsection{Principle of measurement}

The measuring principle of $3 \mathrm{~d}$ laser scanning system is mainly divided into four aspects: ranging, Angle measurement, scanning and orientation. Laser ranging is a key component of laser scanning technology, which plays an important role in positioning laser scanning and obtaining three-dimensional spatial information ${ }^{[3]}$. At present, the main ranging methods are pulse method and phase method.

Take a 3D laser scanner using the pulse ranging method as an example, and the $3 \mathrm{~d}$ laser point coordinate calculation method using the pulse ranging method is shown in Figure 2. The 3d laser scanner obtains the ranging observation value $\mathrm{S}$ by pulse ranging method, and the precision clock control encoder synchronously measures the observed value and zenith distance of each laser pulse transverse scanning Angle. Three-dimensional laser scanning measurement generally uses the internal coordinate system of the instrument. The $\mathrm{X}$ axis is within the transverse scanning surface, the $\mathrm{Y}$ axis is perpendicular to the $\mathrm{X}$ axis, and the $\mathrm{Z}$ axis is perpendicular to the transverse scanning surface. Thus, the calculation formula of $3 d$ laser foot point $P(x, y, z)$ can be obtained (Formula 1) :

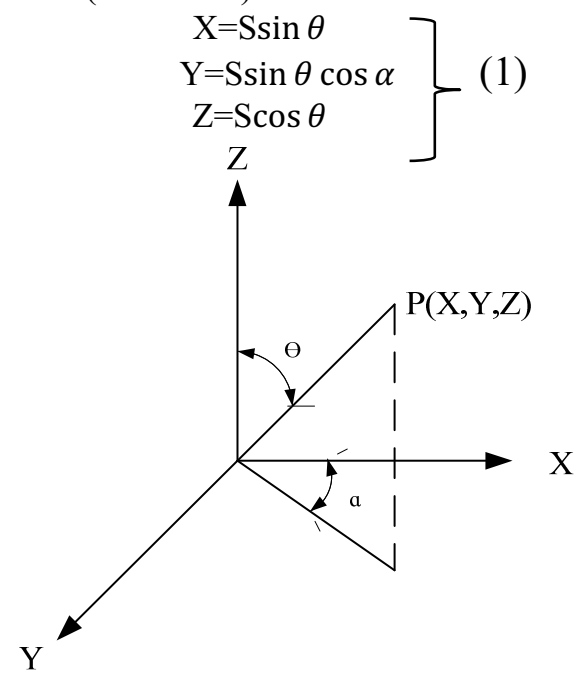

FIG. 2 3D laser point coordinates using pulse ranging method

After the scanner completes a section measurement, the upper part of the scanner rotates the platform and will rotate clockwise or counterclockwise around the vertical axis at a small Angle $\Delta \partial$ to initialize the next section measurement. In this way, the profile scanning measurement is repeated, and multiple profiles are connected to form a scanning block. A complete one often requires multiple scans from different locations to get a complete picture of the solid surface. In order to achieve accurate merging of multiple scanning blocks at different locations, different scanning blocks (point clouds) are required to have certain overlap at the junction. The final point cloud data is transformed into a unified geodetic coordinate, and the software system carries out $3 \mathrm{~d}$ modeling.

\subsection{Classification}

3D scanner can be divided into contact 3D scanner and non - contact 3D scanner. Among them, non-contact 3D scanner is divided into laser scanner and raster 3D scanner (also known as photo $3 \mathrm{~d}$ tracker). And the raster $3 \mathrm{~d}$ scanning and white light scanning or blue light scanning, laser scanner and a bit of laser, line laser, surface laser difference.

At present, there are various kinds of 3D laser scanners in domestic and foreign factories. Generally, they can be divided into three types from the perspective of the spatial position of laser scanning and the operating platform of the scanning system:

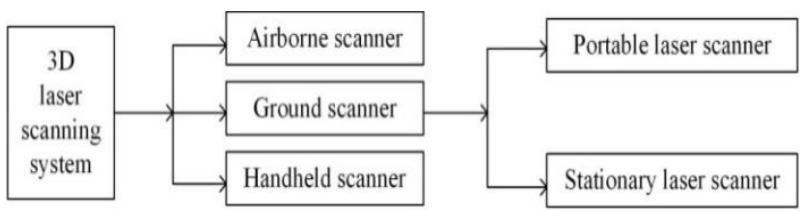

FIG. 3 Divided by scanning space position and operating platform Angle

The ground 3D laser scanner can be divided according to the following three ways:

According to the emission mode of scanner laser beam (as shown in Figure 4) :

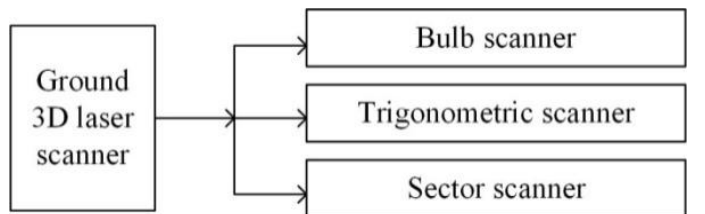

FIG. 4 Distribution by launching mode (Ground 3D laser scanner)

Ground 3D laser scanner is divided according to the system imaging mode (as shown in Figure 5) :

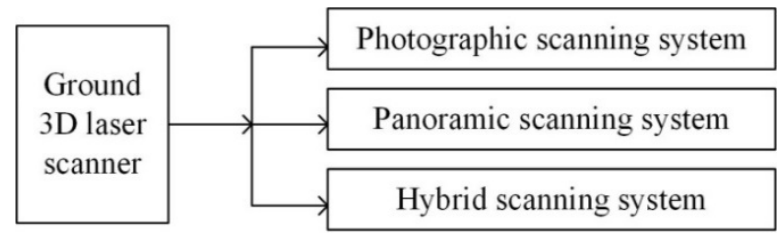

FIG. 5 Imaging mode

According to the scanning system ranging principle (as shown in Table 1): 
Table 1 Divided based on the ranging principle

\begin{tabular}{|c|c|c|}
\hline $\begin{array}{c}\text { Principle of scanning } \\
\text { and ranging }\end{array}$ & range $(\mathrm{m})$ & $\begin{array}{c}\text { data accuracy } \\
(\mathrm{mm})\end{array}$ \\
\hline $\begin{array}{c}\text { Time measurement } \\
\text { (pulse) }\end{array}$ & $\sim 1000$ & $>10$ \\
\hline phase measurement & $<100$ & $<10$ \\
\hline Optical triangle & $<10$ & $<1$ \\
\hline
\end{tabular}

\section{Industrial engineering applications of 3D scanners}

The 3D scanner is another new breakthrough in surveying and mapping technology after GPS space positioning system ${ }^{[4]}$. It can quickly and efficiently obtain the three-dimensional image data of the measured target, which enables surveying and mapping technicians to break through the traditional methods of measuring data processing and enter into the research of new data mining and development. At present, it has been widely used in many fields ${ }^{[5]}$ :

\subsection{Ground landscape shape survey}

Ground landscape shape measurement can provide services for 3d digital design, 3D measurement and reverse engineering, rapid mold manufacturing and other related technologies. It can quickly and accurately complete complex ancient architecture structure measurement, large-scale landscape 3D digital design and template production. The ground shape measurement is shown in FIG. 6.

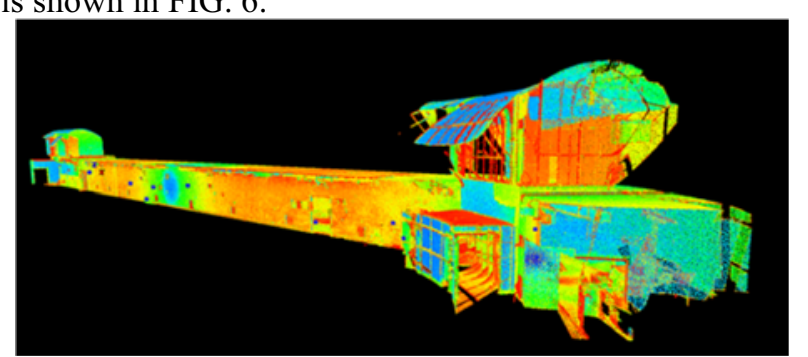

FIG. 6 A ground body survey

\subsection{Measurement and modeling of complex industrial equipment}

In many factories, the pipes and pipes are criss-crossed and of different shapes. It is difficult to find the same point by photogrammetry, which is inefficient, and other traditional methods are even more impotent. And section scanning by laser scanner, get on a multipoint complex three-dimensional point cloud data of industrial equipment, then the point cloud data of different site by data pretreatment and gross error, splicing, merge and application based on the public point of corresponding software can generate the complex model of industrial equipment, to provide equipment manufacturing and plant planning visualization of $3 \mathrm{~d}$ model reference, greatly improve the working efficiency. The scan diagram of a factory is shown in Figure 7.

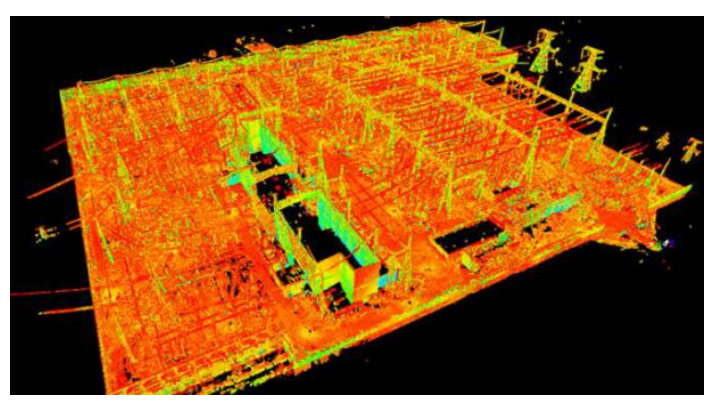

FIG. 7 Schematic diagram of a factory scan

\subsection{Protection of buildings and cultural relics}

Photogrammetry used to be the mainstay, but now prefers to be done with laser scanners. The electronic documents made in this way are easy to preserve, can understand the surface in detail, and can get contour lines, sections and sections conveniently at any time. Provide timely and accurate restoration and recovery data when buildings and artifacts are damaged.

\subsection{Establishment of three-dimensional urban visual model}

Three-dimensional scanning is carried out on the inside and outside of buildings on the street. The scanned point cloud data is processed by data pretreatment, elimination of gross errors, splicing and merging, and ground elevation data and ground object data are obtained by using data filtering and classification algorithm (combined with aerial photography when necessary). The ground elevation data is used to build a high-precision digital ground model. The ground object data can be extracted and processed to realize the establishment of three-dimensional model of the city, and it is also applicable to GIS database update, tour guide and virtual reality production, etc.

\subsection{Banded topographic mapping and mine surveying}

This has previously been done primarily with ground photogrammetry, total station and GPS RTK. The same can now be done quickly and efficiently with a scanner. Namely section scanning the local strip of wild terrain area, repeat scanning in a certain area, joining together, merge $3 \mathrm{~d}$ measurement data, generate images of strip, and then by measuring the amount of control point coordinates of transition to the country or city, with the terrain and object model of three-dimensional point cloud data to establish generated ribbon topographic map, is mainly used for line (railways, roads, rivers, etc.) on both sides of local irregular strip topography measurement. It can also be used in the mine topography, volume, collapse scale and other measurements. A banded terrain scan is shown in Figure 8. 


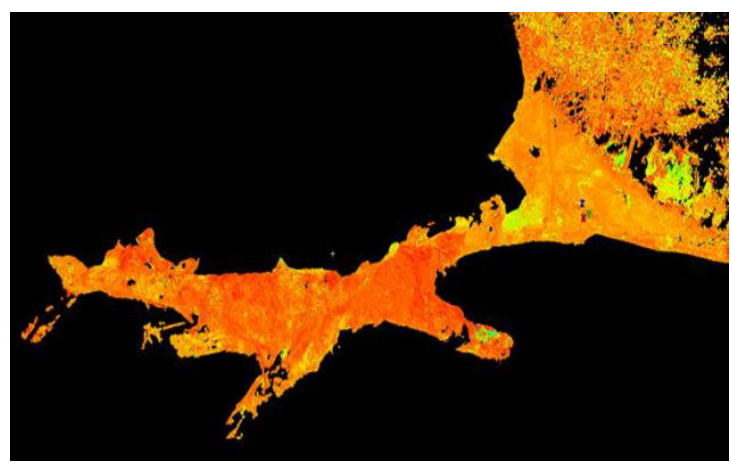

FIG. 8 Banded topographic survey

\subsection{Forest and agricultural resources Survey}

By using laser scanner to scan the trees in the forest, it is possible to obtain more accurately the important parameters (curvature, felling ability, etc.) that can only be estimated or approximately measured by traditional methods. It is also possible to understand the current situation of the forest at a certain moment very accurately. The comparison of measurement results at different times can also be used to understand the forest dynamic changes. Other information can be obtained, such as determining soil quality by measuring tree branches and estimating carbon dioxide content; The measurement data can also obtain the digital ground model and so on.

\subsection{Deformation monitoring}

The ground laser scanner used in deformation monitoring, mainly in the following two aspects: (1) remote ground laser scanner used in landslides, rockfalls, avalanches, mines collapse and other dangerous and difficult to reach the deformation monitoring and calculation, can effectively monitor its range and magnitude of change, applied in disaster prevention and mitigation. The medium - range ground laser scanner is mainly used for deformation measurement of DAMS, locks and Bridges.

\subsection{Medical and industrial measurements}

The scanner used in this field is characterized by short range $(<4 \mathrm{~m})$ and high ranging accuracy $(<1 \mathrm{~mm})$. It applied to online quality control, industrial design, plastic surgery, anthropometry, corrective surgery, etc.

\subsection{Tunnel acceptance}

Aiming at the particularity of tunnel engineering construction, the tunnel measurement into the problems of surveying and calculation of quantity of tunnel excavation, the ideal and the actual mining tunnels between the dig or comparison, and how to quickly get data to ensure that dangerous environment field personnel security in life, such as $3 \mathrm{~d}$ laser scanning technology in the tunnel construction acceptance of the application of solved, save a lot cost for tunnel construction, reduce the risk of field coefficient, provide the best solution for the tunnel construction acceptance. The tunnel scanning case is shown in Figure 9.

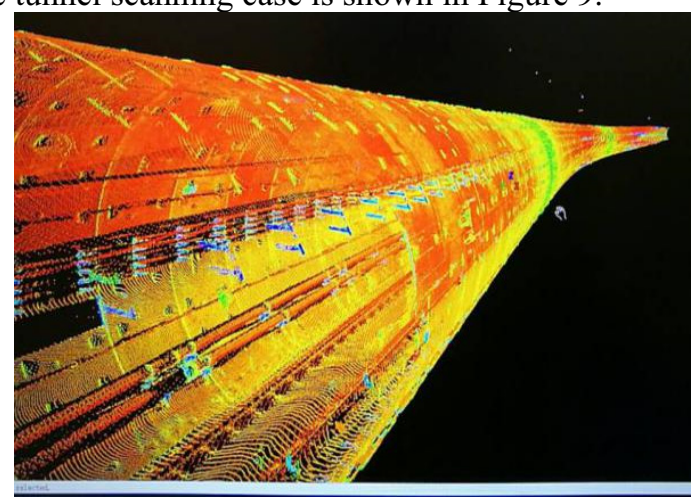

Fig.9 Schematic diagram of tunnel scanning

\section{Conclusion}

As a new measurement method, 3D laser scanner has the following advantages compared with traditional measurement methods: Fast speed, saving a lot of time, complete and accurate measurement; There is no need to touch objects, and darkness and night time do not affect field measurement; Especially suitable for measuring complex objects and their details on the surface; Quickly and accurately determine the surface, volume, section, section, contour, etc. This kind of advanced surveying and mapping technology was first developed in foreign countries and has been widely used in various foreign projects after nearly 20 years of development. And 3D laser scanning technology in our country and the application range compared with abroad still has very big improvement space, thus promoting the technology application has a broad market space, resulting in a considerable economic benefits, at the same time also can drive the technical level of 3D laser scanner, continue to improve, and reached the international advanced level.

\section{Reference}

1. Wang J., Li Z.. Mine Surveting .47:79-83(2019).

2. JJF 1406, Calibration Specification for Terrestrial Laser Scanners (2013).

3. Yan L., Dong M., Liu Y., Chen C., Liu H.. Journal of Geomatics. 44:1-7(2019).

4. Fei Yetai. Error theory and Data Processing. Machinery Industry Press, Beijing (2017).

5. Yang S., Zhang K., Shao Y.. Infrared and Laser Engineering, 1:0106007.1-6(2019). 